\title{
Effect of pregnancy on packed cell volume and hemoglobin level among sudanese pregnant women attending antenatal care at omdurman military hospital (khartoum state).
}

\author{
Mohamed Abdalla Shareef Ahmed ${ }^{1}$, Ibrahim AAli ${ }^{2}$, Omer A Musa ${ }^{2 *}$ \\ ${ }^{1}$ Department of Physiology, Faculty of Medicine, Ibn Sina University, Khartoum, Sudan \\ ${ }^{2}$ Department of Physiology, Faculty of Medicine, The National Ribat University, Khartoum, Sudan
}

\begin{abstract}
Introduction: During pregnancy women undergo several structural and physiological changes almost in most body systems including the hematological profile. The main reported hematological change is plasma expansion and hemodilution which affect the red blood cells (RBCs) and hemoglobin ( $\mathrm{Hb})$ and other indices including packed cell volume (PCV).

Objectives: The objectives of this study were to assess the effect of pregnancy on PCV and $\mathrm{Hb}$ among Sudanese pregnant women and derive a formula for the normal values in pregnant women in relation to the packed cell volume.

Methods: An analytical case control study was conducted in Khartoum state, Sudan 2017. The study included 10 Sudanese pregnant women not taking tonics, 20 Sudanese pregnant women taking tonics attending antenatal care at Omdurman Military hospital and 20 Sudanese nonpregnant women in the same age group as control. A questionnaire was used to collect sociodemographic and clinical data. Blood sample was collected and Complete blood count (CBC) was measured by using automated cell counter Sysmex.
\end{abstract}

Results: The study population comprised 50 women, 10 pregnant women not taking tonics, 20 pregnant women taking tonics and 20 non-pregnant women as control; The results showed a non-significant statistically decrease in $\mathrm{PCV}$ in women not taking tonic $(0.220)$ when compared to pregnant taking tonics and non-pregnant. There was also non-significant statistically decrease in $\mathrm{Hb}$ in pregnant women not taking tonic compared to pregnant taking tonics and non-pregnant with P values (0.104).

Conclusion: During pregnancy there was statistically decrease in PCV values, and count and by considering the hemodilution factor there was a significant increase in values in pregnant ladies not taking tonics.

Keywords: Pregnancy, Hemodilution, PCV, Hemoglobin.

Accepted on September 06, 2018

\section{Introduction}

During pregnancy, the total blood volume increases by about $1.5 \mathrm{~L}$. The plasma volume is increase by $25 \%-80 \%$, reaching its maximum by mid pregnancy. Red cell mass also increases by $10 \%-20 \%$ but the net result is that hemoglobin $(\mathrm{Hb})$ concentration falls. Typically, this is by $1-2 \mathrm{~g} / \mathrm{dL}$ by the late second trimester. Women who take iron supplements have less pronounced $\mathrm{Hb}$ changes, (the increase is approximately $30 \%$ over pre-pregnancy values). It is hard to define a normal reference range for $\mathrm{Hb}$ during pregnancy and the limit for diagnosing anemia. The World Health Organization has suggested that anemia is present in pregnancy when $\mathrm{Hb}$ concentration is $<11 \mathrm{~g} / \mathrm{dL}$. However, large studies in healthy Caucasian women taking iron supplements from mid pregnancy found $\mathrm{Hb}$ values in the early third trimester to be $10.4-13.5 \mathrm{~g} / \mathrm{dL}\left(2.5^{\text {th }}-97.5^{\text {th }}\right.$ centiles $)$. Red cell count and hematocrit (Hct) values are like- wise lower in pregnancy, but the other red cell indices change little. The red cells show more variation in size and shape than in the non-pregnant state.
Hemoglobin and hematocrit increase after delivery. Significant increases have been documented between measurements taken at 6-8 weeks postpartum and those at 4-6 months postpartum, demonstrating that this length of time is needed to restore them to non-pregnant values [1]. Hemoglobin levels fall in pregnancy as a result of a physiological increase in plasma volume that is greater than the pregnancy-associated increase in red cell mass. Iron stores are exhausted by the end of pregnancy in the majority of women unless iron is given. Iron deficiency accounts (IDA) for over $90 \%$ of anemia during pregnancy, therefore iron should be the main-stay of therapy. Anemia affects quality of life and virtually all organs. Maternal anemia in influences mortality, fetal growth, premature death in utero, and fetal programming. Anemia is screened for in pregnancy at booking, 28 weeks and possibly 36 weeks (if the 28 -week test result is low). The frequency of testing is dependent on the country of care France, for example, carries out a hemoglobin level at every pregnancy visit. Anemia screening is primarily done by 
Citation: Ahmed MAS, Ali IA, Musa OA. Effect of pregnancy on packed cell volume and hemoglobin level among sudanese pregnant women attending antenatal care at omdurman military hospital (khartoum state). Gynecol Reproduct Endocrinol -UK. 2018;2(3):1-3

measuring the hemoglobin. Further investigation is usually a ferritin level or a trial of iron. All tests assessing iron status have to be assessed in the light of gestational changes. Oral iron is the usual $1^{\text {st }}$-line treatment in iron deficiency. Parenteral forms of iron are of use if there is non- tolerance or non-compliance of oral iron [1]. Clinical management of hemoglobin in pregnancy is complicated by normal physiological hemodilution, and complicated further by the effects of inflammation on iron metabolism, especially in populations with a high prevalence of obesity or infection. A study in New Zealand area resulted in a total of $46 \%$ of 186 women, with hemoglobin testing at booking, did not have ferritin tested; 86\% (of 385) of ferritin tests were not concurrently tested with $\mathrm{C}$-reactive protein. Despite midwives prescribing iron for $48.7 \%$ of second trimester women, $47.1 \%$ still had low iron status before birth. Only $22.8 \%$ of women had hemoglobin testing postpartum. There was a significant difference between third trimester median ferritin levels in women with $\mathrm{BMI} \geq 25.00(14 \mu \mathrm{g} / \mathrm{L})$ and $\mathrm{BMI}<25.00$ $(18 \mu \mathrm{g} / \mathrm{L})$ [2]. A study on relationship between iron deficiency and thyroid function was done in Chinese women during early pregnancy. The results showed that pregnant women in the mild iron deficiency MID and iron deficiency anemia IDA groups have higher TSH and lower FT4 status than those in the NC group $(p<0.01)$, and the difference between the IDA group and MID group is significant $(p<0.05)$ [3]. In a meta-analysis of $17^{\text {th }}$ studies on maternal anemia during pregnancy and infant low birth weight with a total sample size of 245407 demonstrated that the relative risk for maternal anemia in the first, second and third trimester of pregnancy were 1.26 (95\% CI: $1.03-$ 1.55), 0.97 (95\% CI: 0.57-1.65), and 1.21 (95\% CI: 0.84-1.76), respectively. The relationship between maternal anemia and infant low birth weight in the first trimester of pregnancy was significant [4]. A cross sectional study that elaborates excess menstrual bleeding and interpregnancy interval were statistically associated with anemia among the pregnant women, in study in southern Ethiopia [5]. There are opportunities for increased education on IDA in American College of Obstetricians and Gynecologists, specifically with respect to risk factors. There also appears to be substantial practice variance regarding screening and supplementation for IDA, which may correspond to variability in professional guidelines. Increased education on IDA, especially the importance of sociodemographic factors, and further research and effort to standardize guidelines is needed [6]. As the plasma where increases during pregnancy the hematocrit (Hct) is expected to decrease, due to increase in RBCs and WBCs counts it might not. If the (Hct) decrease then the hemoglobin level is expected to be physiologically low. There for this study will reviewing the prevalence of anemia among Sudanese pregnant ladies in Khartoum state-Sudan and is it truly anemia or there is correct level of hemoglobin by Packed cell volume.

\section{Methods}

This was an analytical case control study conducted at Omdurman Military Hospital, Khartoum, Sudan 2017 to assess the effect of pregnancy on PCV and hemoglobin among Sudanese women. The study included 10 Sudanese pregnant women not taking tonics, 20 Sudanese pregnant women taking tonics attending antenatal care at Omdurman Military hospital and 20 Sudanese non-pregnant women in the same age group as control at the same age group. A questionnaire was used to collect the sociodemographic and clinical data including age, parity, trimester of pregnancy, receive tonics or blood during current pregnancy. Consent was obtained from each individual participating in the study. $5 \mathrm{ml}$ of venous blood collected from each participant by a disposable needle in a container containing EDTA, complete blood count $(\mathrm{CBC})$ was measured using automated cell counter Sysmex. Data were analyzed using the Statistical Package for Social Sciences (SPSS) computer program version 20 ( $\mathrm{P}$ value $\leq 0.05$ considered significant).

\section{Results}

The study of population comprised 50 women, 10 pregnant women not taking tonics, 20 pregnant women taking tonics and 20 non-pregnant women as control; All of them were within the child bearing age. From the analysis, mean values for the pregnant women hemoglobin not using tonics were as follow: $11.34 \pm 0.51$ compared to PCV 35.450 .87 , and the mean values for pregnant women hemoglobin taking tonics $12.11 \pm 0.30$ compared to PCV $37.43 \pm 0.68$, and for women in control group hemoglobin $12.49 \pm 0.27$ compared to PCV $37.13 \pm 0.67$ in control.

The results showed a statistically decrease in PCV in women not taking tonic with $\mathrm{p}$ value $(0.220)$ when compared to pregnant taking tonics and non-pregnant. There is also a statistically decrease in $\mathrm{Hb}$ in pregnant women not taking tonic compared to pregnant taking tonics and non-pregnant with $\mathrm{P}$ values (0.104). When we considered the haemodilution effect on $\mathrm{Hb}$ by applying the equation: $\left\{\frac{\text { plasma(Pregnant })}{\text { Plasma(control) }} \times H b\right\}$ by this facts $a$ pregnant woman with $\mathrm{Hb}$ of $10 \mathrm{gm} / \mathrm{dl}$ (which by equation the corrected $\mathrm{Hb}$ equal $12.7 \mathrm{gm} / \mathrm{dl}$ ) it will be clear that the $\mathrm{Hb}$ will be within the normal range, and the dilution factor is the issue. And when comparing the total $\mathrm{Hb}$ of a normal lady with $\mathrm{Hb}$ of 14 $\mathrm{gm} / \mathrm{dl} \mathrm{n}$ liter equal $\frac{\mathrm{Hb}}{10(\text { converting tolitre })} \times$ blood volume this will equal to $\frac{14}{10} \times 5000=700 \mathrm{gm} / \mathrm{l}$ and for the pregnant woman of $\mathrm{Hb}$ of 10 , (by considering a hemodilution factor $=12.7$ ), the total $\mathrm{Hb}$ level will be $\frac{12.7}{10} \times 6500=($ blood volume of pregnant women $) 635 \mathrm{gm} / \mathrm{l}$

and this will also consolidate my result (Figures 1 and 2).

\section{Discussion}

Pregnancy is a physiological state that is characterized by different hematological changes. In this study we assessed the changes of PCV and hemoglobin count among Sudanese pregnant women in comparison to Sudanese non-pregnant values. The main effect is decrease of PCV and increase of plasma volume during pregnancy that lead to haemodilution.

During pregnancy plasma volume found to be increased, and PCV decreased so if we considered the difference in plasma percentage among pregnant and non-pregnant women and the effect of haemodilution to determine the actual values of $\mathrm{Hb}$ during pregnancy (whom not taking tonics) the results showed a mean $\mathrm{Hb}$ of $11.34 \times 10^{3} / \mathrm{uL}$, when we compared it to the estimated $\mathrm{Hb}$ values during pregnancy the results showed that the mean $\mathrm{Hb}$ value for pregnant women was more relevant to 
Citation: Ahmed MAS, Ali IA, Musa OA. Effect of pregnancy on packed cell volume and hemoglobin level among sudanese pregnant women attending antenatal care at omdurman military hospital (khartoum state). Gynecol Reproduct Endocrinol -UK. 2018;2(3):1-3

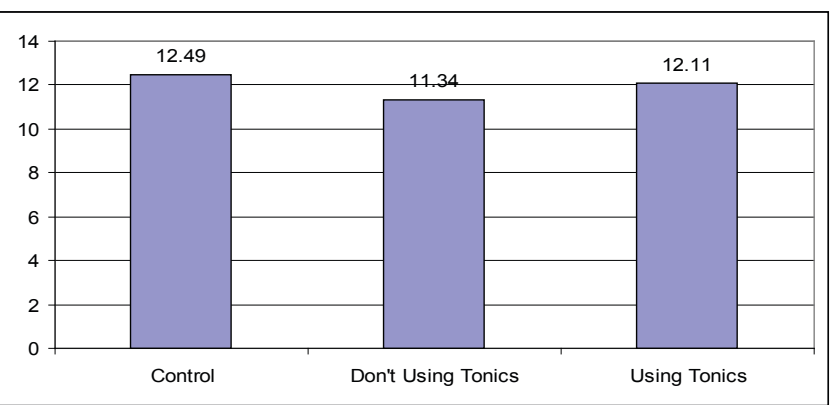

Figure 1: Mean of Hb for control, pregnant taking tonics and pregnant not taking tonics.

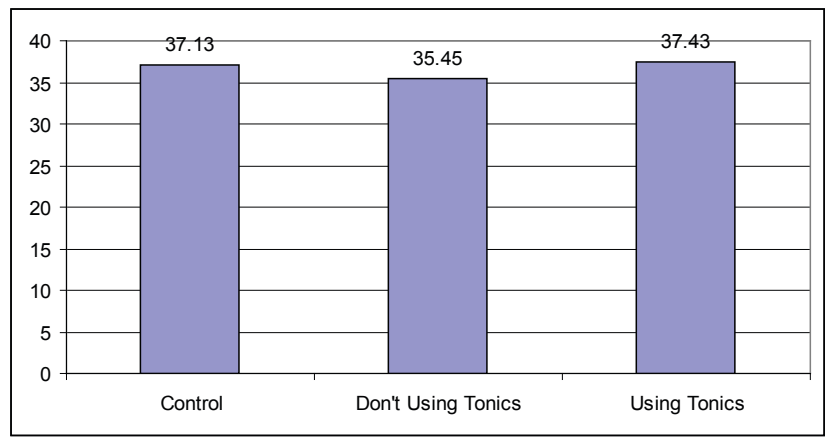

Figure 2: Mean of PCV for control, pregnant taking tonics and pregnant not taking tonics.

the value that calculated by the equation. These results might be due to the massive and rapid increase in plasma volume during pregnancy to compensate the high demand.

\section{Conclusion and Recommendation}

This study concluded that during pregnancy there was decrease in $\mathrm{PCV}$ values and $\mathrm{Hb}$ in comparison to non-pregnant and by considering the haemodilution factor there was a significant relation between $\mathrm{Hb}$ and the calculated one. It is highly recommended to involve $\mathrm{PCV}$ in the routine hematological blood test during pregnancy to consider the haemodilution factor that affects all blood cells.

\section{References}

1. Obstetrics hematology manual. Cambridge University Press, New York, USA, 2010.

2. Calje E, Skinner J. The challenge of defining and treating anemia and iron deficiency in pregnancy: A study of New Zealand midwives' management of iron status in pregnancy and the postpartum period. Birth 2017;44:181-90.

3. Li S, Gao X, Wei Y, et al. The relationship between iron deficiency and thyroid function in Chinese women during early pregnancy. Nutr Sci Vitaminol 2016;62:397-401.

4. Rahmati S, Delpishe A, Azami M, et al. Maternal anemia during pregnancy and infant low birth weight: A systematic review and meta-analysis. Int J Reprod Biomed (Yazd) 2017;15:125-34.

5. Getahun W, Belachew T, Wolide AD. Burden and associated factors of anemia among pregnant women attending antenatal care in southern Ethiopia: Cross sectional study. BMC Res Notes 2017;10:276.

6. Marcewicz LH, Anderson BL, Byams VR, et al. Screening and treatment for iron deficiency anemia in women: Results of a survey of obstetrician-gynecologists. Maternal Child Health J 2017;21:1627-33.

\section{*Correspondence to:}

Omer Abdel Aziz Musa

Faculty of medicine

The National Ribat University

Sudan

E-mail: omusa56@yahoo.co.uk 